\title{
A New MIMO Technique Utilizing Superimposed Auxiliary Signals for Simultaneously Achieving Spatial Multiplexing and Diversity Gains in MIMO-Aided Communication Systems
}

\author{
SADIQ IQBAL ${ }^{1}$, SHAHEER KHALID ${ }^{2}$, JEHAD M. HAMAMREH ${ }^{3}$ \\ ${ }^{1}$ Department of Electrical and Computer Engineering, Antalya Bilim University,Antalya, Turkey (e-mail: sadiq.iqbal@std.antalya.edu.tr) \\ ${ }^{2}$ Department of Electrical and Computer Engineering, Antalya Bilim University,Antalya, Turkey (e-mail: shaheer.khalid@std.antalya.edu.tr) \\ ${ }^{3}$ Department of Electrical and Electronics Engineering, Antalya Bilim University,Antalya, Turkey (e-mail: jehad.hamamreh@antalya.edu.tr) \\ Corresponding author: Sadiq Iqbal (e-mail: sadiq.iqbal@std.antalya.edu.tr) Shaheer Khalid (e-mail: shaheer.khalid@std.antalya.edu.tr)
}

All the codes used to generate the results in this paper can be found at researcherstore.com

\begin{abstract}
Future wireless communication systems demand superior spectral efficiency, enhanced system reliability, improved SNR gain, and better employment of power resources than currently deployed systems. In this work, we propose a new MIMO communication technique that can simultaneously provide multiplexing gain and diversity gain as well as reduce complexity and processing at the receiver side. The proposed system transceiver structure utilizes superimposed auxiliary signals that are designed based on the wireless channel characteristics to eliminate inter antenna interference as well as completely remove the channel effects at the receiver while providing low reception complexity. The efficacy and novelty of the proposed system are verified via extensive mathematical analysis and validated by numerical simulations. The obtained results indicate that the proposed new model achieves high throughput, enhanced reliability, improved connectivity, less complexity, and more efficient communication which is suitable for low power consumption and limited processing applications.
\end{abstract}

INDEX TERMS Multiple Input Multiple Output, Conventional MIMO, Spectral Efficiency, Reliability, 6G, Internet of Things, Physical Layer Technology.

\section{INTRODUCTION}

A ccompanied by global advancements in wireless technology, present-day networks are facing high traffic, maximum reliability and vast coverage demands [1]. To accommodate such demands a new wireless communication system is required, which should lead towards the conceptualization of advanced wireless communication technology ( $5 \mathrm{G}$ and beyond). The major hype of $5 \mathrm{G}$ is due to its three main characteristics that include Ultra-Reliable Low Latency Communication (URLLC), Enhanced Mobile Broadband (eMBB), and Massive Machine Type Communication (mMTC). With these enhanced services and features, 5G communication systems have become more powerful. It will have a remarkable impact on several areas of life, which can support many interesting applications such as smart city projects, smart energy networks, remote surgery, drone delivery, self-driven transportation, virtual reality, and many others [2]. By the passage of time, the paradigm related to $5 \mathrm{G}$ has shifted from the need for a wireless network with a higher data rate towards ultrareliable and low latency communication. $5 \mathrm{G}$ could not offer what it is being marketed for due to new emerging services such as extended reality (XR) that harbors augmented, mixed, and virtual reality to services like telemedicine, haptic, brain-computer interfaces, and connected autonomous systems. To support services like these a wireless system has to simultaneously provide high reliability, low latency, and high data rates with zero interference at the same time [3]. To overcome such challenges a new revolutionary system is needed such as the future $6 \mathrm{G}$ wireless network because such a system is inherently 
designed to be tailored to meet the performance requirements of IoE applications and their accompanying technological trends that require diversification, high data rates, low error rates, and interference-free communication. To achieve all of the above requirements a system must be designed that must meet the criteria of combining all the mentioned benefits [3],

There are many systems present in the literature but out of all these MIMO systems are the most suitable and compatible to be used in 5G, 6G, and beyond. Because MIMO systems have become an integral part of current wireless systems, and especially in recent years they have been used extensively to achieve high spectral efficiency and energy efficiency. However, these new technologies are not enough to accommodate the ever-increasing demands. The wireless users have increased exponentially in the last few years, and these users generate trillions of data that must be handled efficiently with more reliability and security. Additionally, there are billions of IoT devices that have various applications like smart healthcare, smart homes, and smart energy, that contribute to the data traffic. Therefore, it becomes even more apparent why MIMO is the best choice [1].

MIMO systems are arbitrary wireless communication systems, that are considered as a link in which the transmitting end, as well as the receiving end, is equipped with multiple antenna elements. The core paradigm in MIMO systems is space-time signal processing in which time is coupled with the spatial dimension that integrates multiple spatially distributed antennas. Each transmitting antenna in a MIMO system operates on the same frequency and does not require extra bandwidth [4].

To quench the hunger for data-driven applications, the data rates must be increased. It can be achieved by using the technique called spatial multiplexing. In this technique, the data stream is divided (multiplexed) and transmitted into several branches and transmitted via several (independent) channels in space and different bits are transmitted via different antennas, this is achieved by utilizing the multiple paths and effectively using them as additional channels to carry data such that the receiver receives multiple data at the same time. By using spatial multiplexing the data rates are increased but as a result, there is a reduction of bit error rate [5].

Achieving higher data rates at the cost of bit error rate reduces system performance, so to tackle this problem a simple technique known as alamouti space-time block coding (STBC) is used which focuses on increasing transmit diversity [6]. This scheme can improve signal quality at the receiver side and provide better performance of error rate of wireless communication systems, which is done by simple processing at the transmission side between two antennas. But the prerequisite here is that this technique causes the data rate to be the same as in SISO, with no increase in data rate at all. When considering the current huge demand for higher data rates this technique is not desirable at all [6].

All the aforementioned techniques have their merits and demerits as they are used separately in the MIMO system, but to increase the data rate, achieve signal diversity, avoid interference and channel effects completely, the mentioned MIMO techniques are not suitable to be used separately due to related drawbacks. In the literature so far, some papers have been presented that talk about combining these three techniques with MIMO such as [7]. Physical layer design techniques require simple signal processing methods. This is beneficial to services with limited processing and low power requirements [8]. To eliminate interference PLT is used, which requires the channel conditions between the transceivers [9]. In [10], authors use precoder matrices that are the function of wireless channels between the transceiver antennas. The matrices are directly multiplied with the user data before transmission.

Based on the aforementioned discussion, there is a vast requirement for a new and robust MIMO technique utilizing PL for enhanced performance, that can effectively merge and aggregate different advantages provided by individual MIMO schemes such as multiplexing, space-time block coding to efficiently meet the desired qualities like superior throughput, better reliability, efficient utilization of resources, enhanced communication and low complexity. This work proposes a new MIMO paradigm, where, utilization of superimposed auxiliary signals using diversity and multiplexing gains is used to enhance throughput efficiency, reliability, interference elimination, and coverage for future low-complexity, mMTC devices. The main objectives and contributions of the proposed MIMO design utilizing PLT are listed below.

\section{A. NOVELTY AND CONTRIBUTIONS OF THE PROPOSED ALGORITHM}

The novelty and contributions of the proposed MIMO paradigm is to develop a multi-service MIMO scheme with:

1) The ability to combine different schemes in one single technique.

2) Low-power consumption.

3) Simultaneous auxiliary-signal-base transmission for a single user.

4) Low complexity: Conventional MIMO systems use different interference cancellation techniques [11] such as channel inversion and dirty paper coding at the user's receiver to cancel the interference. However, the proposed algorithm uses specially designed auxiliary signals to automatically cancel the interference by simplifying transmission complexity.

5) - Minimum computation: The channel matrices are diagonal, therefore, the inverse operation is simple. Consequently, the auxiliary signal matrices can be designed by simple computation.

The remainder of this work is organized as follows: Section II provides a review of MIMO. Section III discusses the overall system model of the proposed system. The algorithm is discussed in detail in section IV. Section V highlights the simulation results, and finally, the conclusion is presented in section VI. 
TABLE 1. Novelty of the Proposed Model

\begin{tabular}{ll}
\hline \hline Proposed System Model & Literature \\
\hline \hline Single User Scenario & Multi User Scenario \\
\hline One Tx and Rx & Multiple of Tx's and Rx's \\
\hline Single User Link & Multi User Link \\
\hline $\mathbf{x}_{\mathbf{1}}$ and $\mathbf{x}_{\mathbf{2}}$ for a Single User & $\mathbf{x}_{\mathbf{1}}$ for User-1, $\mathbf{x}_{\mathbf{2}}$ for User-2 \\
\hline Both Tx and Rx have two Antennas & Each User has one Antenna \\
\hline Use of Auxiliary Signals & Use of different techniques \\
\hline Less complex & More complex \\
\hline Suitable for IoT devices & Not suitable for IoT devices \\
\hline \hline
\end{tabular}

\section{A MIMO REVIEW}

As wireless media is becoming the most dominant access type, so far most internet-based services, especially in the case of IoT, where millions of devices are connected to the internet, this number will increase in the future. But it raises the concern of designing wireless systems that can be utilized in such devices.

In general, the MIMO systems can provide increased capacity to handle multiple users. But MIMO systems fell short where there is a need for high throughput with system reliability and quality of communication. This critical flaw needs to be taken care of for future wireless systems. All the techniques [5] [6] [12] have their merits and demerits as they are used separately in the MIMO system, but to increase the data rate, achieve signal diversity, avoid interference and provide desired communication experience, MIMO system alone is not enough even with using these three techniques separately with MIMO still leave behind the drawbacks related to each of them. In the literature so far, some papers have been presented that talk about combining these three techniques with MIMO such as [13]

In [14], authors perform a detailed analysis of high-level modulation such as MPSK in a spatial multiplexing MIMO scheme that investigates the effects of AWGN and Rayleigh channel on the BER of high data rates. These analyses are performed only on spatial multiplexing highlights the factors affecting BER. In [6], authors present a simple transmission diversity scheme that solely focuses on improving the system reliability by using two transmission antennas and one receiver antenna. In this scheme, the data rate remains the same only BER is improved.

Authors in [15], propose a new STBC technique designed for a multi-user MIMO system containing two users, that transmit independently, the transmission matrix is designed to maximize coding gain for the two users. The proposed STBC enables independent decoding of symbols of both users and a pairwise maximum likelihood decoder which is derived from the proposed technique. Although, this scheme performs better than the one proposed in [6] but still cannot provide a high data rate as it only focuses on reliability.

In [16], a high rate space-time transmission scheme is proposed that contains codes that are linear in space and time, hence the name is linear dispersion codes (LDC). LDC offers a quite flexible tradeoff between space-time coding and spa- tial multiplexing, the concept applied in this scheme involves a matrix-based linear modulation framework, where each of the space-time transmission matrices is generated using dispersion matrices and the transmitted symbol vectors that determine the component weights. This technique manages to achieve a striking balance between diversity and spectral efficiency but it is still not as efficient in improving the data rate conventional MIMO scheme does.

In [17], a special class of MIMO is presented that is termed as V-BLAST which is similar to Bell Labs Layered Space and Time scheme (BLAST), this scheme aims to increase the system throughput in terms of the number of bits per symbol that are transmitted in a given bandwidth at given integrity but in this scheme, multiple of antennas are activated for a single user.

In [18], authors presented a new transmission scheme that employs 2 STBC layers such that the transmitter has four antennas while the receiver has two antennas. This scheme is known as double space-time transmit diversity (D-STTD) and when comparing with conventional MIMO schemes this scheme outperforms MIMO.

In [19], the authors proposed the technique known as layered steered space-time spreading (LSSTS), to allow multiple users to communicate with each other with the combined benefits of V-BLAST, STS, and beamforming with multicarrier direct sequence CDMA (MC DS-CDMA). The LSSTS employs four transmit antennas and two receive antennas with a linear receiver to decode the received signal.

Therefore, to provide diversity gain, multiplexing gain, and interference-free communication the conventional MIMO schemes proposed in the literature are not suitable for the current wireless communication system. Specifically, for IoT applications and a massive increase in users with the huge demand for high data rate, high reliability, and also to provide the best performance, interference has to be eliminated, due to using many layers and decoders at the receiver side, the complex processing done is also a critical problem because IoT devices require low complexity, power saving, and processing.

In the subsequent sections, a novel MIMO-inspired communication technique is modeled, where the technique combines the various MIMO schemes, but also utilizes characteristics of the channel to provide perfect interference cancellation without any additional processing at the receiver.

\section{OVERALL SYSTEM MODEL}

The proposed communication technique can be modeled to serve any number of users. However, the scheme discussed in this work is designed for a single user for simplicity purposes, whereas the system is composed of two antennas at the transmitter and two antennas at the receiver. The two transmitter antennas simultaneously transmit the same signal to two receiver antennas through different channels as shown in Fig. $1 . \mathbf{H}_{\mathbf{k m}}$ is the diagonal channel frequency response of user $k$ and $m$ antenna during the transmissions. The channels between transmitter antennas and receiver antennas 


\section{Conventional System}

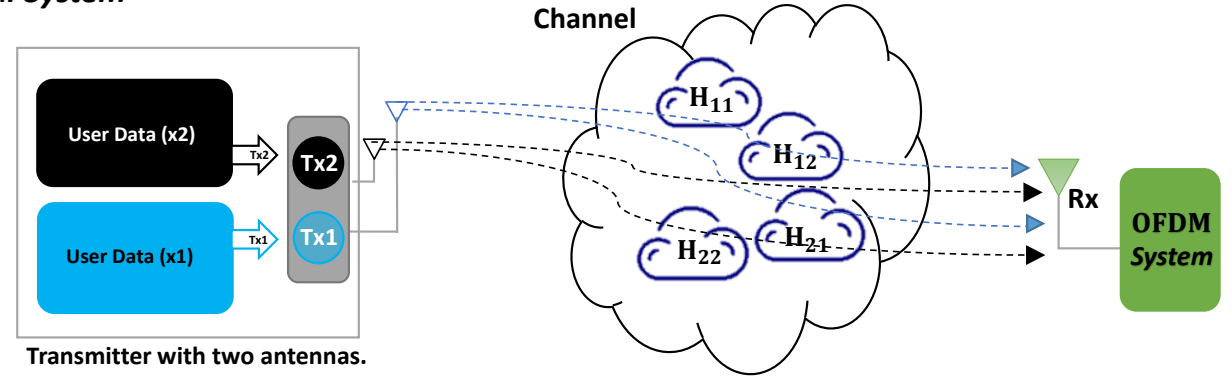

\section{Proposed Model}

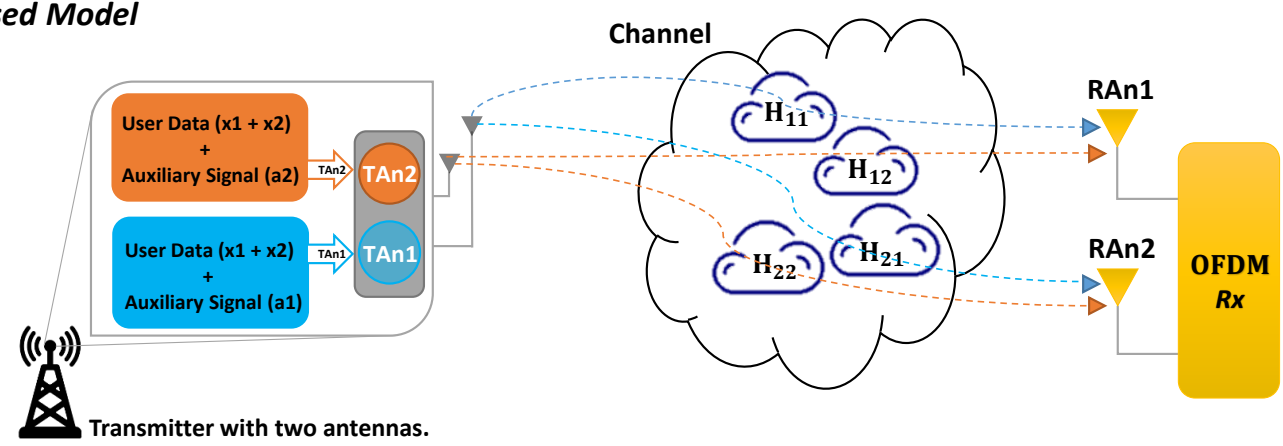

FIGURE 1. Figure illustrating the Conventional and Proposed System Model.

are assumed to be known at the transmitter and are taken to be slowly varying multi-path Rayleigh fading with the exponentially decaying channel.

Moreover, we employ channel-sounding techniques to derive the channels from the transmitter to the receiver. The technique enables the reproduction of the channels using the receiver to transmitter channels in a time division duplexing (TDD) system. The proposed paradigm utilizes two antennas for transmission and superimposed auxiliary signals.

\section{PROPOSED ALGORITHMS}

In this section, we focus on deriving the proposed algorithm and justifying the calculations. This work explores the use of auxiliary signals that are superimposed on user data to enhance reliability and eliminate the interference of future applications with limited processing abilities at the receiver. The system is designed in such a way that there are two simultaneous transmissions from two different antennas with two auxiliary signals $\left(\mathbf{a}_{1}\right.$ and $\left.\mathbf{a}_{2}\right)$ that are calculated and superimposed on top of user data signals during each transmission. The downlink transmission from two different antennas is to ensure different channels. Consequently, enabling the design of the auxiliary signals to guarantee interference-free, reliable, and multiplexed communication with minimal utilization of resources. This is because the designed auxiliary signals are the function of channels both receiver antennas.

The design of the proposed algorithm is as follows: A dual multi-carrier downlink system with two antennas at the transmitter is used, as shown in Fig. 2. Moreover, a single receiver with two antennas is included in the system. The transmission process consists of two transmitter antennas TAn1 and TAn2 that simultaneously transmit the same signal as can be observed in Fig. 1. The frequency response of each OFDM symbol for the receiver at RAn1 and RAn2 can be represented as $\mathbf{x}_{1}=\left[\mathbf{x}_{\mathbf{0}}, \mathbf{x}_{1}, \ldots, \mathbf{x}_{\mathbf{N}_{\mathbf{f}}-\mathbf{1}}\right]$ and $\mathbf{x}_{\mathbf{2}}=\left[\mathbf{x}_{\mathbf{0}}, \mathbf{x}_{1}, \ldots, \mathbf{x}_{\mathbf{N}_{\mathbf{f}}-\mathbf{1}}\right]$ respectively. Where $N_{f}$ is the total number of modulated symbols in one OFDM block, and both $\mathbf{x}_{\mathbf{1}}$ and $\mathbf{x}_{\mathbf{1}} \in C^{\left[N_{f} \times 1\right]}$.

Afterwards, $\mathbf{x}_{\mathbf{1}}$ and $\mathbf{x}_{\mathbf{2}}$ are converted from serial to parallel and are added together. Further, the designed auxiliary matrices are also added to the sum of user symbols before transmission. The design steps of the auxiliary matrices for the proposed model are outlined in the subsequent discussions.

The transmitted superimposed signal from TAn1 is given as:

$$
\mathbf{u}_{1}=\mathbf{x}_{1}+\mathbf{x}_{2}+\mathbf{a}_{1}
$$

Similarly, the transmitted superimposed signal from TAn2 can be given as:

$$
\mathbf{u}_{2}=\mathbf{x}_{1}+\mathbf{x}_{2}+\mathbf{a}_{2}
$$




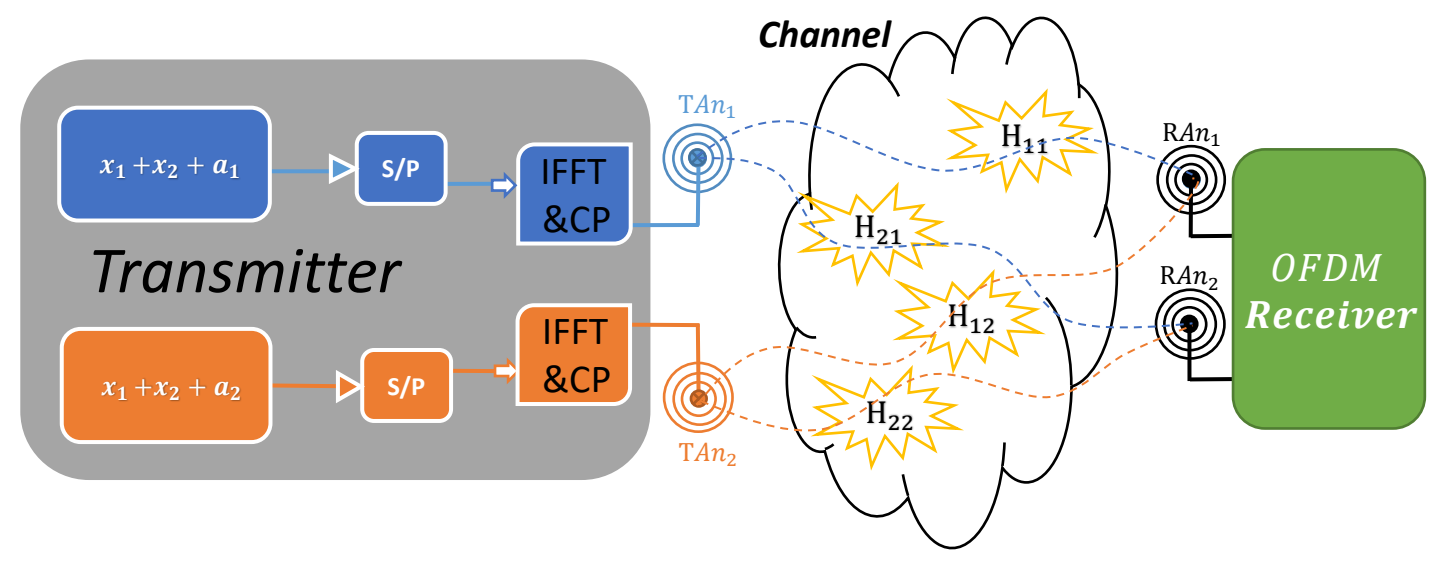

FIGURE 2. Block Diagram of the Proposed MIMO System with Superimposed Auxiliary Signals.

where, $\mathrm{x}_{1}$ and $\mathrm{x}_{2}$ are the vector data in frequency domain intended for RAn1 and RAn2 respectively. Moreover, $\mathbf{a}_{1}$ and $\mathbf{a}_{2}$ are the auxiliary matrices expressly designed using the legitimate receiver's channel. $\mathbf{a}_{1}$ and $\mathbf{a}_{2}$ will make sure that the RAn1 and RAn2 will get reliable signals which are also protected from interference. In the following subsections, we will explain the details of the received signals at RAn1 and RAn2. Afterward, we will explain the design of the auxiliary signals.

\section{A. RECEIVED SIGNAL AT RECEIVER ANTENNA-1 (RAN1)}

The received signal in the frequency domain at RAn1 from transmission through TAn1 can be given as:

$$
\mathbf{y}_{11}=\mathbf{H}_{11} \mathbf{u}_{1},
$$

where $\mathbf{H}_{11}$ is the frequency response of the channel between RAn1 and TAn1. Similarly, the received signal at RAn1 from transmission using TAn2 is given as:

$$
\mathbf{y}_{12}=\mathbf{H}_{12} \mathbf{u}_{2},
$$

where $\mathbf{H}_{12}$ is the frequency response of the channel between RAn1 and TAn2. The combined received signal at RAn1 from transmission through TAn1 and TAn2 can be given as:

$$
\hat{\mathbf{y}}_{1}=\mathbf{y}_{11}+\mathbf{y}_{12}+\mathbf{z}_{1},
$$

where $\mathbf{y}_{11}$ is the received signal at RAn1 from TAn1, $\mathbf{y}_{12}$ is the received signal at RAn1 from TAn2 and $\mathbf{z}_{1} \mathrm{~s}$ the additive white gaussian noise (AWGN) at RAn1. After substituting the values of $\mathbf{y}_{11}$ and $\mathbf{y}_{12}$ in (5), the combined signal is written as follows:

$$
\hat{\mathbf{y}}_{1}=\mathbf{H}_{11} \mathbf{u}_{1}+\mathbf{H}_{12} \mathbf{u}_{2}+\mathbf{z}_{1},
$$

where $\mathbf{u}_{1}$ and $\mathbf{u}_{2}$ are the superimposed transmitted signals. After substituting the values of $\mathbf{u}_{\mathbf{1}}$ and $\mathbf{u}_{\mathbf{2}}$ in (6), the combined signal is:

$$
\hat{\mathbf{y}}_{1}=\mathbf{H}_{11}\left(\mathrm{x}_{1}+\mathrm{x}_{2}+\mathbf{a}_{1}\right)+\mathbf{H}_{12}\left(\mathrm{x}_{1}+\mathrm{x}_{2}+\mathbf{a}_{2}\right)+\mathbf{z}_{1} .
$$

Rearranging (7) and collecting like terms gives us:

$$
\begin{aligned}
\hat{\mathbf{y}}_{1}=( & \left.\mathbf{H}_{11}+\mathbf{H}_{12}\right) \mathbf{x}_{1}+\left(\mathbf{H}_{11}+\mathbf{H}_{12}\right) \mathbf{x}_{2} \\
+ & \mathbf{H}_{11} \mathbf{a}_{1}+\mathbf{H}_{12} \mathbf{a}_{2}+\mathbf{z}_{1} .
\end{aligned}
$$

The first term in (8) is the desired term for RAn1, while the remaining terms are undesired. The interference cancellation auxiliary signals will ensure that the undesired terms, as well as the channel effects, are removed and canceled at RAn1.

\section{B. RECEIVED SIGNAL AT RECEIVER ANTENNA-2 (RAN2)}

Similar to RAn1, the received signal in the frequency domain at RAn2 using TAn1 is given as:

$$
\mathbf{y}_{21}=\mathbf{H}_{21} \mathbf{u}_{1},
$$

where $\mathbf{H}_{\mathbf{2 1}}$ is the frequency response of the channel between RAn2 and TAn1. Likewise, the received signal at RAn2 using TAn2 is given as:

$$
\mathbf{y}_{22}=\mathbf{H}_{22} \mathbf{u}_{2},
$$

where $\mathbf{H}_{22}$ is the frequency response of the channel between RAn2 and TAn2. The combined received signal at RAn2 from transmission through TAn1 and TAn2 can be written as:

$$
\hat{\mathbf{y}}_{2}=\mathbf{y}_{21}+\mathbf{y}_{22}+\mathbf{z}_{2},
$$


where $\mathbf{y}_{\mathbf{2 1}}$ is the received signal at RAn2 from TAn1 and $\mathbf{y}_{\mathbf{2} 2}$ is the received signal at RAn2 from TAn2 and $\mathbf{z}_{2}$ is the AWGN at the RAn2. After substituting the values of $\mathbf{y}_{21}$ and $\mathbf{y}_{22}$ into (11), the combined signal is presented as follows:

$$
\hat{\mathbf{y}}_{2}=\mathbf{H}_{21} \mathbf{u}_{1}+\mathbf{H}_{22} \mathbf{u}_{2}+\mathbf{z}_{2},
$$

where $\mathbf{u}_{1}$ and $\mathbf{u}_{2}$ are the superimposed transmitted signals. Substituting the values of $\mathbf{u}_{1}$ and $\mathbf{u}_{2}$ into (12) results in the combined signal shown below.

$$
\hat{\mathbf{y}}_{2}=\mathbf{H}_{21}\left(\mathrm{x}_{1}+\mathrm{x}_{2}+\mathbf{a}_{1}\right)+\mathbf{H}_{22}\left(\mathrm{x}_{1}+\mathrm{x}_{2}+\mathbf{a}_{2}\right)+\mathbf{z}_{2} .
$$

Rearranging (13) and collecting like terms gives us:

$$
\begin{aligned}
\hat{\mathbf{y}}_{2}=( & \left.\mathbf{H}_{21}+\mathbf{H}_{22}\right) \mathbf{x}_{1}+\left(\mathbf{H}_{21}+\mathbf{H}_{22}\right) \mathbf{x}_{2} \\
& +\mathbf{H}_{21} \mathbf{a}_{1}+\mathbf{H}_{22} \mathbf{a}_{2}+\mathbf{z}_{2},
\end{aligned}
$$

The second term in (14) is the desired term for RAn2 while the remaining terms are undesired. Likewise, the superimposed auxiliary signals will make sure that the undesired terms, as well as the channel effects, are removed and canceled at RAn2.

\section{DESIGNING THE SUPERIMPOSED AUXILIARY SIGNALS}

In this section, we will design the auxiliary signals $\mathbf{a}_{\mathbf{1}}$ and $\mathbf{a}_{2}$ such that the signals sent during each transmission are received at the intended receiver with no extra computations required while providing interference-free, reliable, and much higher quality communication. The design of auxiliary signals is inspired by [9], [10], [20]. In [10], the precoders $\mathbf{P}_{1}, \mathbf{P}_{2}, \mathbf{P}_{3}$, and $\mathbf{P}_{4}$ are designed using the wireless channel characteristics and multiplied with the user data to eliminate interference and channel effects. However, the auxiliary signals $\mathbf{a}_{1}$ and $\mathbf{a}_{2}$ are superimposed on top of user data before transmission.

The auxiliary signals are designed as follows: As illustrated in (8), the first term is the desired term for receiver-1. Therefore, the auxiliary signals $\mathbf{a}_{1}$ and $\mathbf{a}_{2}$ will be designed in a way that the effect of the channels on RAn1 is removed along with the interference. Hence, the undesired second, third, and fourth term in (8) should be equated to zero as follows:

$$
\left(\mathbf{H}_{11}+\mathbf{H}_{12}\right) \mathbf{x}_{2}+\mathbf{H}_{11} \mathbf{a}_{1}+\mathbf{H}_{12} \mathbf{a}_{2}=0 .
$$

Similarly, looking at (14), the second term of (14), is the desired term for RAn2. Therefore, the auxiliary signals $\mathbf{a}_{\mathbf{1}}$ and $\mathbf{a}_{2}$ will be designed such that the effect of the channels on RAn2 is removed as well as the interference caused by RAn1 on RAn2. Hence, the first, third, and fourth term in (14) should be equal to zero and can be shown as:

$$
\left(\mathbf{H}_{21}+\mathbf{H}_{22}\right) \mathbf{x}_{1}+\mathbf{H}_{21} \mathbf{a}_{1}+\mathbf{H}_{22} \mathbf{a}_{2}=0 .
$$

Equations (15) and (16) can jointly be solved to determine the values of auxiliary signals $\mathbf{a}_{1}$ and $\mathbf{a}_{\mathbf{2}}$ as follows:

$$
\mathbf{F}=\mathbf{H}_{12}-\mathbf{H}_{11} \mathbf{H}_{21}{ }^{-1} \mathbf{H}_{22},
$$

$$
\mathbf{a}_{2}=\mathbf{F}^{-1}\left(\left(\mathbf{H}_{11}+\mathbf{H}_{11} \mathbf{H}_{21}{ }^{-1} \mathbf{H}_{22}\right) \mathbf{x}_{1}-\left(\mathbf{H}_{11}+\mathbf{H}_{12}\right) \mathbf{x}_{2}\right),
$$

$$
\mathbf{a}_{1}=-\left(\mathbf{I}+\mathbf{H}_{21}{ }^{-1} \mathbf{H}_{22}\right) \mathbf{x}_{\mathbf{1}}-\mathbf{H}_{21}{ }^{-1} \mathbf{H}_{22} \mathbf{a}_{2} .
$$

The values of auxiliary signals $\mathbf{a}_{1}$ and $\mathbf{a}_{\mathbf{2}}$ obtained from equations (18) and (19) will be used in simultaneous transmission of superimposed user data to get highly reliable, spectral efficient, and interference-free signals.

\section{SIMULATION RESULTS}

In this section, we analyze the performance of proposed model. We present the simulation results of the proposed algorithm using bit error rate (BER), throughput error rate (TER), and peak to average power ration (PAPR). The parameters used in this work are depicted in the table (2) below.

TABLE 2. Proposes Algorithm System Parameters

\begin{tabular}{ll}
\hline \hline Channel & Multipath Rayleigh Fading Channel \\
\hline Channel Length & 9 \\
\hline Cyclic Prefix (CP) & 9 \\
\hline FFT Size & 64 \\
\hline Modulation Type & BPSK \\
\hline \hline
\end{tabular}

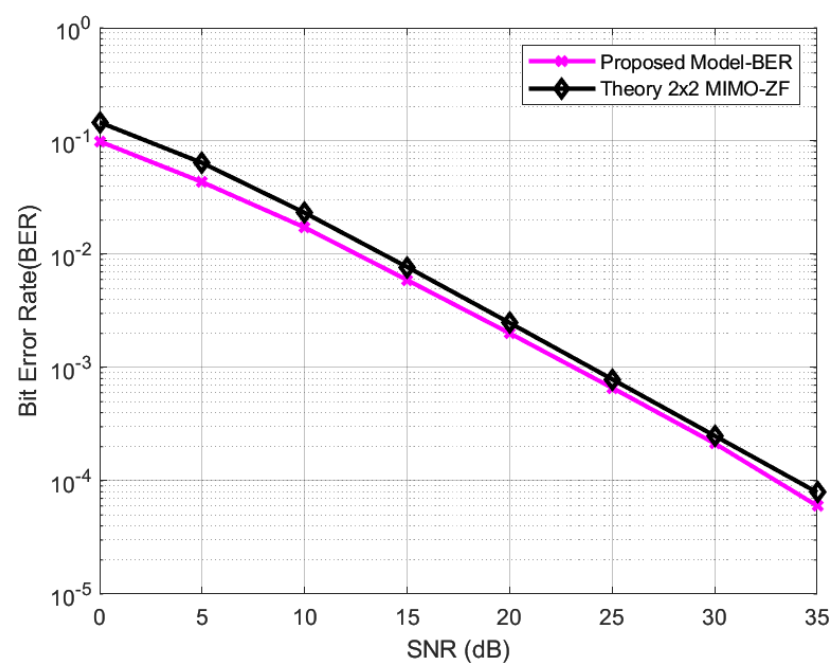

FIGURE 3. BER Vs SNR performance measure for the proposed algorithm

The designed system uses an OFDM transmitter that has TAn1 and TAn2 antennas with 64 sub-carries for each receiver antenna as shown in Fig. 2. In addition, a cyclic prefix (CP) of length 9 is used to prevent inter-symbol interference (ISI). The channel between TAn1 and TAn2, and receiver antennas (RAn1) and (RAn2), is assumed to be multi-path Rayleigh fading channel with equal number of taps $(L=9)$ as shown in table 2 . 
Fig. 3 depicts the BER verses SNR graphs for proposed model BER utilizing the proposed algorithm, and theory $2 \times 2$ MIMO-ZF. From Fig. 3, it can be observed that the proposed model BER performs better than the theory $2 \times 2 \mathrm{MIMO}-\mathrm{ZF}$ BER. This indicates

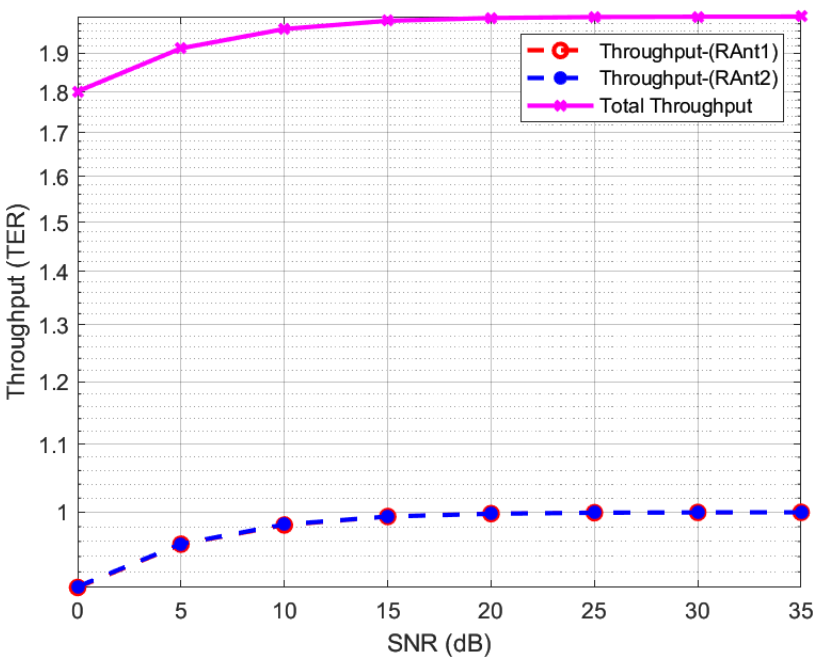

FIGURE 4. Throughput error rate of the proposed algorithm

that the proposed algorithm can provide efficient and reliable communication due to (18) and (19) respectively.

Fig. 4 shows the throughput analysis for a user utilizing the proposed algorithm. From Fig. 4, we can observe that the total throughput performance is twice the TER performance of both RAn1 and RAn2 because the user receives the combined signal. Moreover, this clearly shows that the throughput (data rate) for the user is doubled while retaining the BER performance from Fig. 3.

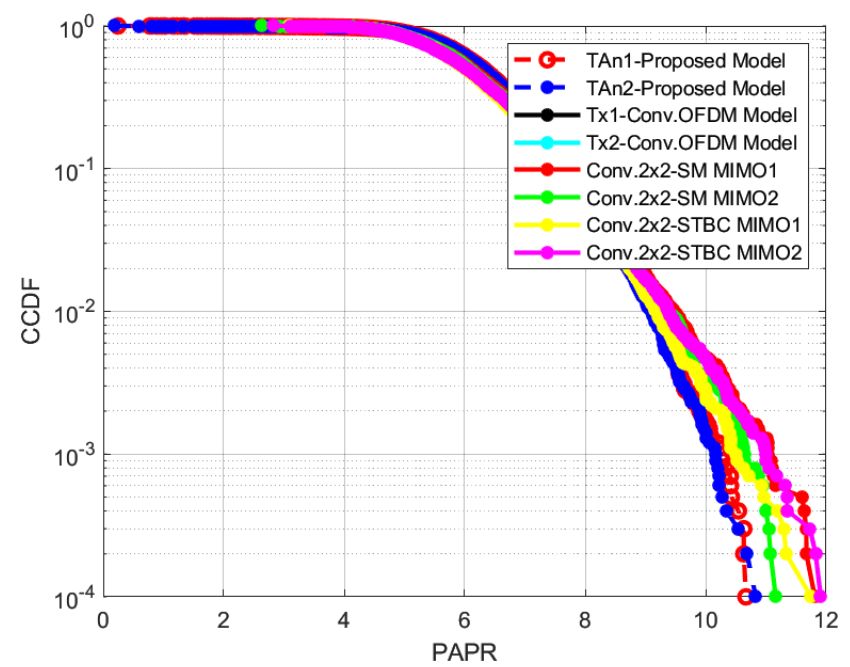

FIGURE 5. Peak to Average Power Ratio (PAPR) of the proposed algorithm.

Fig. 5 depicts the peak to average power ratio (PAPR) of a conventional OFDM, spatial multiplexing, STBC system, and an OFDM system utilizing the proposed algorithm. TAn1-proposed model and TAn2-proposed model are the
PAPR of the RAn1 and RAn2 using the proposed technique, on the other hand, Tx1-conv. OFDM Model and Tx2-conv. OFDM Model are the PAPR of a conventional OFDM system as well as the conventional 2x2 SM MIMO and STBC MIMO systems. Fig. 5 indicates that the user utilizing the proposed algorithm has better PAPR performance than the one's using conventional OFDM, conv. SM, and STBC MIMO. Hence, the proposed system solves one major problem experienced by OFDM and conv. MIMO systems [21], by reducing the PAPR leading to better spectral and energy efficiency.

\section{CONCLUSION}

In this work, we proposed a novel MIMO communication technique that combines various schemes of conventional MIMO systems and has better spectral efficiency, reliability, resilience, effectiveness, and low complexity, without having the receiver to do any additional processing. The scheme is made up of two antennas at the transmitter that simultaneously transmits data. Two distinct channel-dependent auxiliary signals are superimposed on top of transmitted signals from TAn1 and TAn2, one auxiliary signal for each data symbol such that each legitimate receiver gets its intended signal. The paradigm is validated with mathematical models and simulations. The obtained results demonstrate that the proposed system can provide reliable and highly efficient communication with minimum complexity than conventional communication techniques by making the proposed model suitable for IoT applications with low complexity and low power requirements. In the future, we aim to design this communication technique for more than one user with multiple antennas at both transmitter and receiver.

\section{REFERENCES}

[1] R. Chatau, "Massive MIMO systems for 5G and beyond networksoverview, recent trends, challenges, and future research direction." NCBI 2020, vol. 20, no. 10 .

[2] J. M. Hamamreh and Z. M.F., "An advanced non-orthogonal multiple access security technique for future wireless communication networks," RS Open Journal on Innovative Communication Technologies, vol. 1, no. 2, 2020.

[3] M. B. W. Saad and M. Chen, "A vision of $6 \mathrm{G}$ wireless systems: Applications, trends, technologies, and open research problems," IEEE Network, vol. 34, no. 3, pp. 134-142, 2020.

[4] S. B R, "Space-time block coding (STBC) for wireless networks," International Journal of Distributed and Parallel Systems, vol. 3, 2012.

[5] A. Vadhera and L. Kansal, "BER analysis of 2x2 Spatial Multiplexing under AWGN and Rayleigh Channels for Different Modulations," Global Journal of Researches in Engineering Electrical and Electronics Engineering, vol. 13, no. 11, pp. 53-58, 2013.

[6] A. S. M., "A simple transmit diversity technique for wireless communications," IEEE J. Sel. Areas Commun., vol. 16, no. 8, pp. 1451-1458, 2013.

[7] M. El-Hajjar and L. Hanzo, "Multifunctional MIMO systems: A combined diversity and multiplexing design perspective,' IEEE Wireless Communications, vol. 17, no. 2, pp. 73-79, 2010.

[8] J. M. Hamamreh and H. Arslan, "Physical layer security for NOMA requirements, merits, challenges, and recommendations," arXiv:1905.05064, 2019.

[9] J. Lemayian and J. Hamamreh, "A novel small-scale nonorthogonal communication technique using auxiliary signal superposition with enhanced security for future wireless networks," RS Open Journal on Innovative Communication Technologies, 102020 . 
[10] — - "Hybrid MIMO: A new transmission method for simultaneously achieving spatial multiplexing and diversity gains in MIMO systems," RS Open Journal on Innovative Communication Technologies, 52021.

[11] S. Patel, "Over view of interference cancellation techniques in MUMIMO," International Journal on Wireless Optical Communications, vol. 7, pp. 312-316, 102015 .

[12] J. Hamamreh and H. Arslan, "Joint PHY/MAC layer security design using ARQ with MRC and null-space independent, PAPR-aware artificial noise in SISO systems," IEEE Transactions on Wireless Communications, pp. $1-1,2018$.

[13] M. El-Hajjar and L. Hanzo, "Multifunctional MIMO systems: A combined diversity and multiplexing design perspective,," IEEE Wireless Communications,, vol. 17, no. 2, pp. 73-79, 2010.

[14] L. Vadhera, Anuj Kansal, "BER analysis of 2x2 MIMO spatial multiplexing under AWGN and rician channels for different modulations techniques," International Journal of Wireless Mobile Networks, pp. 8598, 2013.

[15] N. Sharma, "Space time block code for next generation multi-user MIMO systems," in Procedia Computer Science, vol. 34. ScienceDirect, 2014, pp. $172-179$.

[16] H. Jafarkhani, "A quasi-orthogonal space-time block code," in IEEE Transactions on Communications, vol. 49. IEEE, 2001, pp. 1-4.

[17] B. Hassibi and B. M. Hochwald, "High-rate codes that are linear in space and time," in IEEE Transactions on Information Theory, vol. 48. IEEE, July 2002, pp. 1804-1824,.

[18] A. G. D. E. N. Onggosanusi and T. A. Schmidl, "High rate space-time block coded scheme: performance and improvement in correlated fading channels," in IEEE Wireless Communications and Networking Conference Record, vol. 1. IEEE, 2002, pp. 194-199.

[19] L.-L. Yang and L. Hanzo, "Performance of generalized multicarrier DSCDMA over nakagami-m fading channels," in IEEE Transactions on Communications, vol. 50. IEEE, 2002, pp. 956-966,

[20] H. J. Lemayian Joel and A. Mohamedou, "New non-orthogonal transmission schemes for achieving highly efficient, reliable, and secure multiuser communications," RS Open Journal on Innovative Communication Technologies, 2020.

[21] I. Baig, N. ul Hasan, M. Zghaibeh, I. U. Khan, and A. S. Saand, "A DST precoding based uplink NOMA scheme for PAPR reduction in 5G wireless network," in 2017 7th International Conference on Modeling, Simulation, and Applied Optimization (ICMSAO). IEEE, 2017, pp. 1-4.

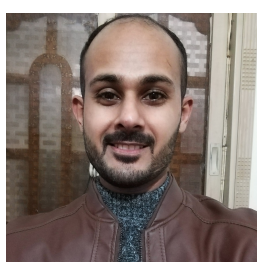

SADIQ IQBAL received a B.E. degree in electrical engineering from QUEST University Nawabshah, Pakistan in 2016. He is presently pursuing a master's (M.Sc.) degree in electrical and computer engineering. He is currently with Antalya Bilim University, Turkey. His research interests include physical layer technology, 5G Communication networks, Artificial Intelligence, Deep Learning, and IoT and its applications.

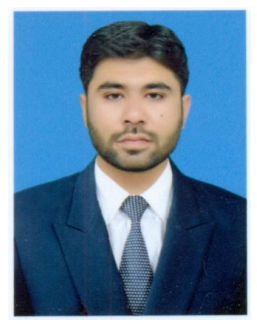

SHAHEER KHALID received a B.Sc. degree in electrical engineering from Government College University Faisalabad, Pakistan. He is presently pursuing a master's (M.Sc.) degree in electrical and computer engineering. He is currently with Antalya Bilim University, Turkey. He is currently working as a research assistant at Antalya Bilim University.

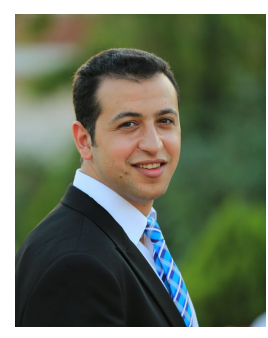

JEHAD M. HAMAMREH received the B.Sc. degree in electrical and telecommunication engineering from An-Najah University, Nablus, in 2013, and the Ph.D. degree in electrical-electronics engineering and cyber systems from Istanbul Medipol University, Turkey, in 2018. He was a Researcher with the Department of Electrical and Computer Engineering, Texas A and M University at Qatar. $\mathrm{He}$ is currently an Assistant Professor with the Electrical and Electronics Engineering Department, Antalya International (Bilim) University, Turkey.

His current research interests include wireless physical and MAC layers security, orthogonal frequency-division multiplexing multiple-input multiple-output systems, advanced waveforms design, multi-dimensional modulation techniques, IoT, 5G \& 6G, and orthogonal/non-orthogonal multiple access schemes for future wireless systems. He is a Regular Reviewer for various refereed journals as well as a TPC Member for several international conferences. 